\title{
Multifractality of wave functions at the quantum Hall transition revisited
}

\author{
F. Evers, ${ }^{1}$ A. Mildenberger, ${ }^{2}$ and A. D. Mirlin ${ }^{1,2, *}$ \\ ${ }^{1}$ Institut für Nanotechnologie, Forschungszentrum Karlsruhe, 76021 Karlsruhe, Germany \\ ${ }^{2}$ Institut für Theorie der Kondensierten Materie, Universität Karlsruhe, 76128 Karlsruhe, Germany
}

(Received 22 August 2001; published 16 November 2001)

\begin{abstract}
We investigate numerically the statistics of wave function amplitudes $\psi(\mathbf{r})$ at the integer quantum Hall transition. It is demonstrated that in the limit of a large system size the distribution function of $|\psi|^{2}$ is log-normal, so that the multifractal spectrum $f(\alpha)$ is exactly parabolic. Our findings lend strong support to a recent conjecture for a critical theory of the quantum Hall transition.
\end{abstract}

DOI: 10.1103/PhysRevB.64.241303

PACS number(s): 73.43.Cd, 71.30.+h, 73.43.Nq

In 1980 von Klitzing, Dorda, and Pepper discovered ${ }^{1}$ that the Hall conductance $\sigma_{\mathrm{xy}}$ of a two-dimensional electron gas develops plateaus at values quantized in units of $e^{2} / h$. Twenty years later, the integer quantum Hall effect (IQHE) still constitutes one of the great challenges of condensedmatter physics. Initially, the effort focused on the physics of the Hall plateaus which is by now fairly well understood. ${ }^{2,3}$ Then interest has shifted towards the transition region, where $\sigma_{\mathrm{xy}}$ crosses over from one plateau to the next. However, here the situation is not as well resolved. It has been understood from early on that this is a second-order phase transition, and the scaling scenario has been confirmed in numerous experiments and computer simulations ${ }^{4}$ yielding, e.g., the value $\nu$ $\simeq 2.35$ for the localization length exponent. In contrast, analytical approaches did not lead to quantitative predictions. The ultimate goal here is to identify the effective low-energy theory of the critical point (expected to be a conformal field theory) and to calculate critical exponents and other characteristics of the transition region. In particular, independence of the critical theory on microscopic parameters would establish universality of the critical exponents which is a matter of intensive and controversial discussions in the experimental literature.

The earliest field-theoretical formulation of the problem was given by Pruisken ${ }^{5}$ (see Ref. 6 for a more precise, supersymmetric version) and has the form of the nonlinear $\sigma$-model with a topological term. Since the latter is invisible in perturbation theory, one has to resort to nonperturbative means in order to address the critical behavior. Pruisken and co-workers were thus led to the dilute instanton gas approximation. ${ }^{7}$ However, this approximation can only be justified in the weak-coupling limit, $\sigma_{x x} /\left(e^{2} / h\right) \gg 1$, and becomes uncontrolled in the critical region $\sigma_{x x} \sim e^{2} / h$. For this reason, no quantitative predictions for critical properties have been made within this approach. Another line of efforts was based on a mapping of the low-energy sector of Pruisken's model onto an antiferromagnetic superspin chain. ${ }^{8}$ The superspin chain was also obtained by starting from the Chalker-Coddington network model of the IQHE. ${ }^{9}$ However, attempts to find an analytical solution of the superspin chain problem remained unsuccessful.

Recently, two papers appeared which may signify a breakthrough in the quest for the conformal critical theory of the IQHE. Zirnbauer ${ }^{10}$ and, a few months later, Bhaseen et al., ${ }^{11}$ proposed that this is a $\sigma$-model with the Wess-ZuminoNovikov-Witten (WZNW) term $\Gamma$,

$$
S[g]=\frac{1}{8 \pi \lambda^{2}} \int d^{2} x \operatorname{Str} \partial_{\mu} g^{-1} \partial_{\mu} g+k \Gamma,
$$

where $g$ belongs to a certain symmetric superspace (see Refs. 10 and 11 for a detailed exposition), and $\lambda$ and $k$ are coupling constants. The theories considered in Refs. 10 and 11 differ only in the value of the constant $k$ in front of the WZNW term, $k=1$ in Ref. 10 and $k=1 / \lambda^{2}$ in Ref. 11. If true, the conjecture of Refs. 10,11 will provide a framework for a systematic study of the IQHE critical behavior. While the exponent $\nu$ has not yet been found on this basis, a nontrivial prediction for the statistics of critical eigenfunctions has been obtained. Specifically, it was found in Ref. 11 that the corresponding multifractality spectrum is exactly parabolic (which means that the distribution of eigenfunction intensities is log-normal),

$$
\widetilde{f}(\alpha)=2-\left(\alpha-\alpha_{0}\right)^{2} / 4\left(\alpha_{0}-2\right),
$$

with $\alpha_{0}-2=2 \lambda^{2}$ (a formal definition of the function $\widetilde{f}(\alpha)$ is given below). For the model of Ref. 10 a parabolic multifractal spectrum of a somewhat different quantity, the twopoint conductance, was obtained. ${ }^{12} \mathrm{We}$ can show, however ${ }^{13}$ that the two statements are closely related, so that the findings of Refs. 10,12 imply again the result (2) for the eigenfunction statistics, with $\alpha_{0}-2=4 \lambda^{2}$. The exact parabolicity (2) of the multifractality spectrum constitutes a stringent prediction, numerical verification of which would be a serious check of validity of the theory proposed in Refs. 10,11. This is the aim of the present paper.

Our interest in this problem was additionally motivated by the fact that the previously published numerical results $3,4,14-17$ appeared to be in clear conflict with the prediction (2), showing strong deviations from parabolicity. Furthermore, it has been a widespread belief that the parabolic approximation cannot in principle be exact, since the singularity spectrum $f(\alpha)$ is only defined on an interval $\alpha_{-}<\alpha$ $<\alpha_{+}$, where it is positive, and has infinite derivatives at the termination points, $f^{\prime}\left(\alpha_{-}\right)=f^{\prime}\left(\alpha_{+}\right)=\infty$. As we demonstrate below, these earlier findings were crucially affected by finite-size effects and by the absence of a proper ensemble averaging. 
To begin with, we recall the general framework used for the description of critical wavefunction statistics. One introduces a set of inverse participation ratios ${ }^{18}$

$$
P_{q}=\int_{L^{d}} d^{d} r|\psi(\mathbf{r})|^{2 q}, \quad q \geqslant 0,
$$

where $\psi(\mathbf{r})$ is the wavefunction amplitude and $L$ the system size. (For the IQHE dimensionality is $d=2$.) These moments show in the large $L$-limit scaling behavior characterized by a set of exponents, which can be defined for both the average and the typical value of $P_{q}$,

$$
\begin{gathered}
\left\langle P_{q}\right\rangle=\tilde{c}_{q} L^{-\tilde{\tau}_{q},} \\
P_{q}^{\mathrm{typ}} \sim \exp \left\langle\ln P_{q}\right\rangle=c_{q} L^{-\tau_{q},}
\end{gathered}
$$

where $\langle\ldots\rangle$ denotes ensemble averaging. As long as $q$ is small enough, $q<q_{c}$, the distribution function of $P_{q}$ is sufficiently narrow and $\tilde{\tau}_{q}=\tau_{q}$. However, with increasing $q$ this distribution function becomes broader. Most importantly, it shows a power-law tail $\propto P_{q}^{-1-x_{q}}$ at large $P_{q}$, where $x_{q}$ decreases with increasing $q$. The critical value $q_{c}$ is determined by the condition $x_{q_{c}}=1$. For $q>q_{c}$ the average $\left\langle P_{q}\right\rangle$ is governed by rare events, and $\tau_{q}>\tilde{\tau}_{q}$. We refer the reader to Ref. 19 for more details.

Instead of using the moments $P_{q}$ one can study directly the distribution $\mathcal{P}$ of the wavefunction amplitudes. It is easy to see that (4) translates, in the limit $L \rightarrow \infty$, into

$$
\mathcal{P}(\alpha) \sim(\ln L)^{1 / 2} L^{-d+\widetilde{f}(\alpha)}, \quad \alpha=-\ln |\psi|^{2} / \ln L,
$$

where the function $\tilde{f}(\alpha)$ is related to the exponents $\tilde{\tau}_{q}$ via the Legendre transformation,

$$
\tilde{\tau}_{q}=q \alpha-\widetilde{f}(\alpha) ; \quad q=\widetilde{f}^{\prime}(\alpha) .
$$

Similarly, one can define the $f(\alpha)$-spectrum of a typical eigenfunction (which satisfies $f(\alpha) \geqslant 0,{ }^{3}$ )

$$
f(\alpha)=q \alpha-\tau_{q} ; \quad q=f^{\prime}(\alpha) .
$$

The two definitions coincide, $f(\alpha)=\widetilde{f}(\alpha)>0,{ }^{18}$ for $q<q_{c}$, or, equivalently, for $\alpha_{-}<\alpha<\alpha_{0}$, where $\alpha_{0}$ describes the scaling of the typical value, $\exp \left\langle\ln |\psi|^{2}\right\rangle \propto L^{-\alpha_{0}}$, and $\alpha_{-}$denotes the zero of $f, f\left(\alpha_{-}\right)=\widetilde{f}\left(\alpha_{-}\right)=0$. On the other hand, in the regime of rare events, $\alpha<\alpha_{-}$, the function $\widetilde{f}(\alpha)$ becomes negative, while $f(\alpha)$ is not defined. The best estimate for $\alpha_{0}$ from previous numerical work is $2.26 \pm 0.01{ }^{16}$ The value of $\alpha_{-}$obtained in Ref. 16 is $1.1 \pm 0.1$, incompatible with (2).

It is important to realize that the field-theoretical prediction (2) refers to the function $\widetilde{f}(\alpha)$, since in the theory one deals with averaged moments (4). In contrast, the earlier numerical studies were devoted to the multifractal spectrum of a single eigenfunction, thus yielding $f(\alpha)$ as an output. We further turn to a common misconception concerning an infinite slope of the $f(\alpha)$ spectrum at its termination point, $f^{\prime}\left(\alpha_{-}\right)=\infty$, which seems to rule out the parabolic form (2). Indeed, in any finite system $\tau_{q}$ must be an analytic function of $q$, implying that $f(\alpha)$ meets the $\alpha$-axis with infinite slope. ${ }^{3}$ This is, however, nothing but an artifact of a finite system size $L$. For any given $L$ there is a finite interval of $\alpha$ close to $\alpha_{-}$where the corresponding spectrum $f_{L}(\alpha)$ deviates from its asymptotic form and approaches the axis with an infinite derivative. In the limit $L \rightarrow \infty$ this interval shrinks to zero, and the spectrum acquires its limiting form $f_{\infty}(\alpha)$ $\equiv f(\alpha)$, corresponding to a nonanalytic $\tau_{q}$,

$$
\tau_{q}= \begin{cases}\tilde{\tau}_{q}, & 0<q<q_{c} \\ q \alpha_{-}, & q>q_{c} .\end{cases}
$$

The situation is similar to a phase transition where singularities also occur in the thermodynamic limit only. Practically speaking, this means that $\tau_{q}$ and $\tilde{\tau}_{q}$, though identical in the thermodynamic limit on the interval $0<q<q_{c}$, have different finite-size corrections.

For calculating the critical wavefunctions at the quantum Hall transition we employ the Chalker-Coddington network model ${ }^{20}$ In order to obtain the wavefunction we translate the lattice dynamics into a unitary time evolution operator $\mathbf{U}$ which describes the wave packet propagation on the network in discrete time steps. ${ }^{16}$ The desired critical wavefunctions are the eigenfunctions of $\mathbf{U}$, which are found by numerical diagonalization.

The calculations were performed for systems of the size ranging from $L=16\left(6 \cdot 10^{6}\right.$ wave functions) to $L=1280$ (2000 wave functions). We implemented efficient numerical packages $^{21-23}$ which allowed us not only to (partly) diagonalize large systems, but also to do it fast enough in order to collect sufficient statistics. Specifically, for a system of size $L$ and for each disorder realization we diagonalized a complex $N \times N$ matrix of the size $N=2 L^{2}$ (which reached $N \approx 3.3$ $\cdot 10^{6}$ for the largest $L$ ) with machine accuracy. For comparison, the biggest realization of the network model reported in the literature that we are aware of is smaller by a factor of five in linear dimensions. ${ }^{16}$ Since an accurate extrapolation to the thermodynamic limit was of primary importance for our work, it was crucial that we could observe the finite size corrections over almost two orders of magnitude in $L$.

As a first test of the validity of Eq. (2), we check its two particular implications, namely $\alpha_{0}-2=2-\alpha_{1}$ and $\alpha_{1 / 2}=2$, where $\alpha_{q}$ is determined by (7), $\alpha_{q}=d \tilde{\tau}_{q} / d q$. We evaluated $\alpha_{q}(L)$ for different system sizes,

$$
\alpha_{q}(L)=-\left\langle|\psi|^{2 q} \ln |\psi|^{2}\right\rangle /\left\langle|\psi|^{2 q}\right\rangle \ln L,
$$

and then extrapolated to the infinite system size, $\alpha_{q}$ $=\lim _{L \rightarrow \infty} \alpha_{q}(L)$, according to

$$
\alpha_{q}(L)=\alpha_{q}+\frac{\lambda_{q}}{\ln L}\left(1+\gamma_{q} \frac{\xi_{\text {irr }}^{y}}{L^{y}}+\cdots\right) .
$$

The most important finite-size effect is of the form $1 / \ln L$ according to (4), with a coefficient $\lambda_{q}=d \ln \tilde{c}_{q} / d q$. The second term in brackets in (11) stems from finite-size corrections to (4) and is governed by the leading irrelevant scaling exponent $y$. The corresponding length scale $\xi_{\text {irr }}$ can be in principle absorbed in the coefficient $\gamma_{q}$. 


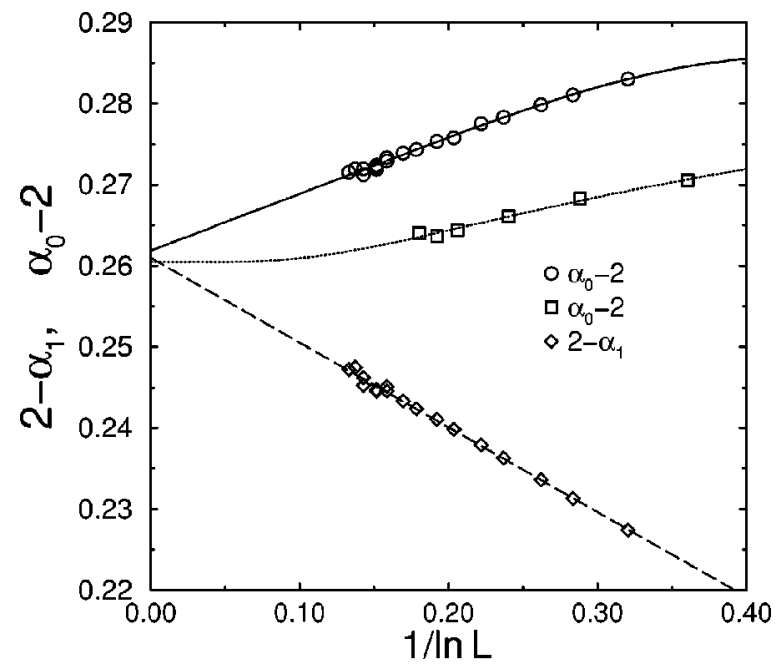

FIG. 1. Finite size effects for the scaling exponents $\alpha_{0}(\bigcirc$, fit solid line) and $\alpha_{1}$ ( $\diamond$, fit dashed line) defined in (10). Also shown is the result for $\alpha_{0}(\square$, fit dotted line) from the transfer matrix calculation, Eq. (12).

The raw data that provides the basis for the extrapolation to $L \rightarrow \infty$ is shown in Fig. 1. From the fit (11) we find $\alpha_{0}$ $=2.261 \pm 0.003, \quad \lambda_{0}=0.09 \pm 0.02$, and $\alpha_{1}=1.739 \pm 0.002$, $\lambda_{1}=-0.11 \pm 0.01$. A similar analysis for the case $q=1 / 2$ yields $\alpha_{1 / 2}-2=0.0001 \pm 0.0002, \lambda_{1 / 2}=-0.042 \pm 0.002$. It is clearly seen that within the accuracy of the numerical data $\alpha_{0}-2$ and $2-\alpha_{1}$ indeed coincide and $\alpha_{1 / 2}-2$ vanishes, in agreement with (2). The irrelevant exponent $y$ is found to be $y=0.4 \pm 0.1$, which is consistent with earlier results ${ }^{4,24}$ (the accuracy of its determination is not very high, since the corresponding finite-size correction is rather small).

As an additional check we have calculated $\alpha_{0}$ in yet another way, which uses a conformal mapping to a quasi-onedimensional strip of the Chalker-Coddington network. It was shown by $\operatorname{Janßen}^{17}$ that $\alpha_{0}$ is related to the localization length $\xi_{L}$ in a strip of width $L$ :

$$
\alpha_{0}-2=(1 / \pi) \lim _{L \rightarrow \infty} L / \xi_{L} .
$$

Since this relation ties $\alpha_{0}$ to the amplitude $\Lambda$ of the scaling law $\xi_{L}=L \Lambda$, the finite-size corrections on the strip take a form different from (10),

$$
\alpha_{0}(L)=\alpha_{0}+\gamma\left(\xi_{\text {irr }} / L\right)^{y}+\cdots .
$$

We have calculated $\xi_{L}$ using the transfer matrix method and found $\alpha_{0}=2.260 \pm 0.003$, in full agreement with the previous result. This confirms the fundamental assumption that the critical theory is conformally invariant. The irrelevant scale index is obtained as $y=0.45 \pm 0.1$, in agreement with earlier findings.

Having satisfied ourselves that numerical results for $\alpha_{0}$, $\alpha_{1 / 2}$, and $\alpha_{1}$ are in favor of the conjecture (2), we turn to an analysis of the $\widetilde{f}(\alpha)$ spectrum in a broader range of $\alpha$. Results of the calculation of $\widetilde{f}(\alpha)$ according to Eq. (6) from the distribution function $\mathcal{P}\left(\left|\psi^{2}\right|\right)$ for different system sizes are shown in Fig. 2. It is seen that $\widetilde{f}(\alpha)$ changes appreciably

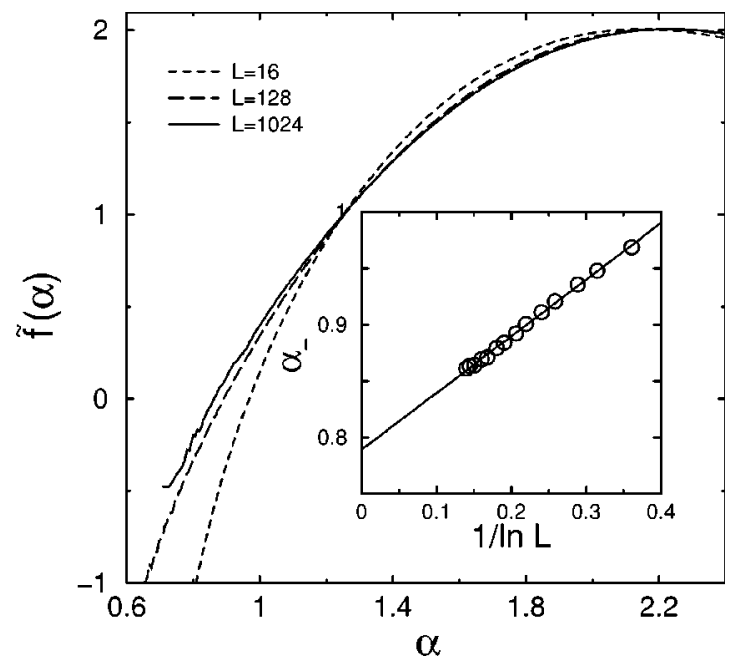

FIG. 2. Evolution of $\widetilde{f}(\alpha)$, as calculated from the distribution function (6), with the system size $L$. The inset shows the corresponding change of $\alpha_{-}$, defined by $\widetilde{f}\left(\alpha_{-}\right)=0$. An extrapolation to infinite $L$ yields: $\alpha_{-}=0.8 \pm 0.02$.

with $L$, which points to the importance of the extrapolation to $L \rightarrow \infty$. In particular, the inset of Fig. 2 shows the evolution of the zero $\alpha_{-}$of $\widetilde{f}(\alpha)$ with $L$. Extrapolating to infinite system size [by taking into account the leading finite-size correction, which has the same $1 / \ln L$ form as in Eq. (11)] yields the value $\alpha_{-}=0.8 \pm 0.02$ in the thermodynamic limit. This agrees with the result $0.816 \pm 0.004$ based on Eq. (2) in combination with the value of $\alpha_{0}$ found above, providing a further strong support to the parabolic law (2).

In order to obtain the whole $\widetilde{f}(\alpha)$ curve in the thermodynamic limit with highest possible accuracy, we return to the procedure based on the evaluation of moments, Eqs. (4) and

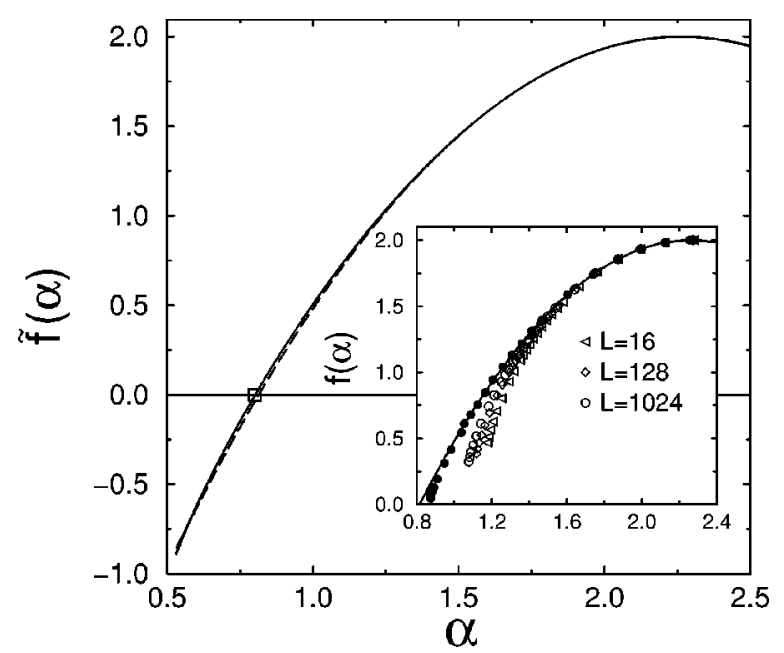

FIG. 3. Multifractal spectrum $\widetilde{f}(\alpha)$ (solid line). The dashed line shows the theoretical conjecture (2) with $\alpha_{0}=2.262$. The point where $\widetilde{f}(\alpha)$ vanishes according to the data presented in Fig. 2 is also shown ( $\square$ ). Inset: spectrum $f(\alpha)$ of a typical eigenfunction for different $L$ and extrapolated to $L \rightarrow \infty$ (O). The line shows the parabolic law. 
(10), since it has the advantage that we have full control over finite-size corrections, Eq. (11) (the transformation from the moments to the distribution function Eq. (6) induces additional corrections proportional to higher powers of $1 / \ln L)$. Performing an extrapolation to $L \rightarrow \infty$, we get the $\widetilde{f}(\alpha)$ curve shown in Fig. 3, which represents the central result of this Rapid Communication. The figure demonstrates a perfect agreement between the obtained $\widetilde{f}(\alpha)$ and the parabolic form (2) with $\alpha_{0}=2.262$. Of course, a numerical analysis can never guarantee that two quantities are identical rather than just very close to each other. However, taking into account that there is no small parameter in the problem, we believe that an accidental closeness of $\widetilde{f}(\alpha)$ to a parabola with such a high accuracy is extremely improbable. We thus conclude that the theoretical prediction (2) stating that the multifractal spectrum is exactly parabolic is correct.

To illustrate the difference and the relation between $f(\alpha)$ and $\widetilde{f}(\alpha)$ and to make a closer contact to earlier works, we show in the inset of Fig. 3 the $f(\alpha)$ spectrum as obtained for different system sizes. The extrapolation to $L \rightarrow \infty$ gives a good agreement with the parabola at $\alpha>\alpha_{0}$, except for a close vicinity of $\alpha_{-}$, where the finite-size corrections to $f(\alpha)$ become especially strong.

Finally, we mention an open issue concerning the values of the coupling constant $\lambda$ and $k$ in Eq. (1) determining $\alpha_{0}$ in the theory. While Zirnbauer ${ }^{10}$ gave convincing arguments in favor of $k=1$, the mechanism of fixation of $\lambda$ remains unclear. In particular, whether or not $\lambda$ is universal is an important open question which requires further analytical and numerical studies.

In conclusion, we have studied the statistics of critical wave functions at the IQHE transition point. We implemented a powerful algorithm which allowed us to reach unprecedented large system sizes and to gather sufficiently good statistics. Having performed the ensemble averaging and an analysis of the finite-size corrections, we calculated the multifractality spectrum $\widetilde{f}(\alpha)$ of critical wave functions in the thermodynamic limit $L \rightarrow \infty$ for $0.5 \leqslant \alpha \leqslant 2.5$. The result is in perfect agreement with the parabolic form (2) with $\alpha_{0}=2.262 \pm 0.003$ and hence it supports the recent conjecture for the form of the conformal field theory of the IQHE critical point. ${ }^{10,11}$

We are thankful to X. S. Li and to the Computer Centers of the Forschungszentrum and the University of Karlsruhe for technical assistance. Discussions with M. Janßen, R. Klesse, D. G. Polyakov, L. Schweitzer, A. M. Tsvelik, P. Wölfle, and M. R. Zirnbauer are gratefully acknowledged. This work was supported by the SFB 195 and the Schwerpunktprogramm "Quanten-Hall-Systeme" der Deutschen Forschungsgemeinschaft.
*Also at Petersburg Nuclear Physics Institute, 188350 St. Petersburg, Russia.

${ }^{1}$ K. von Klitzing et al., Phys. Rev. Lett. 45, 494 (1980).

${ }^{2}$ The Quantum Hall Effect, edited by R. E. Prange and S. M. Girvin (Springer, New York, 1992).

${ }^{3}$ M. Janßen, O. Viehweger, U. Fastenrath, and J. Hajdu, Introduction to the Theory of the Integer Quantum Hall Effect ( $\mathrm{VCH}$, Weinheim, 1994).

${ }^{4}$ B. Huckestein, Rev. Mod. Phys. 67, 357 (1995).

${ }^{5}$ A.M.M. Pruisken, Nucl. Phys. B 235, 277 (1984).

${ }^{6}$ H.A. Weidenmüller, Nucl. Phys. B 290, 87 (1987).

${ }^{7}$ H. Levine et al., Nucl. Phys. B 240, 30 (1984); ibid. 240, 49 (1984); ibid. 240, 71 (1984).

${ }^{8}$ M.R. Zirnbauer, Ann. Phys. (Leipzig) 3, 513 (1994).

${ }^{9}$ D.H. Lee, Phys. Rev. B 50, 10788 (1994); J. Kondev and J.B. Marston, Nucl. Phys. B 497, 639 (1997); M.R. Zirnbauer, J. Math. Phys. 38, 2007 (1997).

${ }^{10} \mathrm{M}$. Zirnbauer, hep-th/9905054v2.

${ }^{11}$ M.J. Bhaseen et al., Nucl. Phys. B 580, 688 (2000).

${ }^{12}$ M. Janßen et al., Phys. Rev. B 59, 15836 (1999).

${ }^{13}$ The connection is established as follows. Using the results of R. Klesse and M. Zirnbauer, cond-mat/0010005, it is easy to show that $\left\langle T_{l m}^{q}\right\rangle \sim L^{d}\left\langle\left|\psi_{m}\right|^{2(1-q)}\left|\psi_{l}\right|^{2 q}\right\rangle$, where $l$ and $m$ are two points of the network, $T_{l m}$ is the corresponding conductance, $\psi_{l}$ and $\psi_{m}$ the wavefunction amplitudes, and $q<1 / 2$. This leads to the following relation between the exponents $X_{q}$ characterizing the scaling of moments of the two-point conductance, $\left\langle T_{l m}^{q}\right\rangle$ $\sim L^{-X_{q}}$, and the indices $\tilde{\tau}_{q}$ defined in (3), $X_{q}=d+\tilde{\tau}_{q}+\tilde{\tau}_{1-q}$. The parabolic form (2) of the wavefunction multifractality leads thus to a parabolic spectrum for the conductance too, $X_{q}$ $=X_{t} q(1-q)$ (which is exactly what was found in Refs. 12,10), with $X_{t}=2\left(\alpha_{0}-d\right)$. The result $X_{t}=8 \lambda^{2}$ of Ref. 10 translates in this context into $\alpha_{0}-2=4 \lambda^{2}$.

${ }^{14}$ B. Huckestein, B. Kramer, and L. Schweitzer, Surf. Sci. 263, 125 (1992).

${ }^{15}$ I. Varga et al., Europhys. Lett. 36, 437 (1996).

${ }^{16}$ R. Klesse and M. Metzler, Europhys. Lett. 32, 229 (1995); Int. J. Mod. Phys. C 10, 577 (1999).

${ }^{17}$ M. Janßen, Int. J. Mod. Phys. B 8, 943 (1994).

${ }^{18}$ We restrict ourselves to $q \geqslant 0$ (corresponding to $\alpha<\alpha_{0}$ ) in this paper. One can generalize the whole consideration to the range of negative $q\left(\alpha>\alpha_{0}\right)$, but this requires some modification of the definition (3).

${ }^{19}$ F. Evers and A.D. Mirlin, Phys. Rev. Lett. 84, 3690 (2000); A.D. Mirlin and F. Evers, Phys. Rev. B 62, 7920 (2000).

${ }^{20}$ J.T. Chalker and P.D. Coddington, J. Phys. C 21, 2665 (1988).

${ }^{21}$ P.R. Amestoy et al., Comput. Methods Appl. Mech. Eng. 184, 501 (2000); P.R. Amestoy et al., SIAM J. Matrix Anal. Appl. 23, 15 (2001).

${ }^{22}$ J.W. Demmel et al., SIAM J. Matrix Anal. Appl. 20, 720 (1999).

${ }^{23}$ R.B. Lehouca, D. Sorensen, and C. Yang, ARPACK Users Guide (SIAM, Philadelphia, 1998).

${ }^{24}$ F. Evers and W. Brenig, Phys. Rev. B 57, 1805 (1998). 